\title{
Developing local services for people with a learning disability and a psychiatric disorder
}

\author{
Ashox Roy, Consultant Psychiatrist; and Stuart Cumella, Senior Research Fellow, \\ University of Birmingham Department of Psychiatry, Queen Elizabeth Psychiatric \\ Hospital, Birmingham B15 2QZ
}

Recent ministerial statements and health circulars have identified a key role for the NHS in providing services for people with a learning disability who have a mental illness or a severe behaviour disorder (NHS Management Executive, 1992). This is not an insignificant task, given that psychiatric disorders (including both mental illness and/or severe behaviour disorders) occur among approximately $30 \%$ of people with a moderate or severe learning disability (Corbett, 1979; Lund, 1985). Patients with psychiatric disorders have proved particularly difficult to resettle from mental handicap hospitals, and form a substantial proportion of the patients who become long-stay residents of mental handicap hospitals despite the development of community-based services. It is therefore essential that each district health authority defines the most appropriate pattern of services for this group of patients, as part of their purchasing strategy for mental health. The type of service required was discussed by the department of Health report Needs and Responses: Services for Adults with Mental Handicap who are Mentally III, who have Behaviour Problems, or who Offend. This noted that no consistent pattern of services has yet emerged, and that suitable alternatives included admission to a specialised mental illness unit in a mental handicap hospital, admission to a general psychiatric ward, admission to a small staffed house, or treatment by a community support team.

This paper reports the progress achieved on one English health region (the West Midlands) in developing services for people with a learning disability who also have a psychiatric disorder. It follows a survey of local services undertaken as part of a comprehensive review commissioned by the West Midlands Regional Health Authority (WMRHA).

\section{The study}

The West Midlands Health Region has a population of 5.2 million people, and comprises 20 health districts. At the time of the survey (August 1991), there were seven mental handicap hospitals which still admitted patients, and a total of 24 consultant psychiatrists in post specialising in the treatment of adult patients with learning disabilities. The consultants were responsible for 25 catchment areas, with a mean population of 208,000 . Most catchment areas corresponded to health district boundaries. A postal questionnaire was sent to each of these consultant psychiatrists, requesting information about the services available to them for treating people with a learning disability both in hospital and in the community. The questionnaire also asked consultants to identify whether a community mental handicap team (CMHT) operated in their catchment area, and the range of professions included in the team.

\section{Findings}

The results of the questionnaire indicated that two of the mental handicap hospitals (admitting patients from 11 of the 25 catchment areas) had a designated ward for admitting people with a learning disability and psychiatric disorder. Two other hospitals (admitting patients from seven catchment areas) had wards nominally designated for the admission of patients with both a learning disability and a psychiatric disorder, but usually had a few vacancies and were therefore frequently blocked to admissions. The remaining three hospitals had no designated admission wards and no wards specifically designated for treating patients with a psychiatric disorder. Patients with psychiatric disorders were admitted to ordinary wards, according to the availability of a vacant bed.

There were also variations between different parts of the region in the provision of community-based services for people with a learning disability and psychiatric disorder. At the time of the survey, 13 catchment areas had access to community-based facilities for this group of patients. Other community-based specialist provision included respite care facilities (seven catchment areas), long-term residential facilities (six catchment areas), day care facilities (seven catchment areas), and specialised teams providing care for individuals with severely disturbed behaviour (five catchment areas). None of the catchment areas in the region had home treatment teams comparable with the programmes which 
exist in mental illness services for the intensive home treatment of patients with acute psychiatric disorders (Dean \& Gadd, 1990).

The distribution throughout the region of hospital and community-based services for people with a learning disability and a psychiatric disorder was very uneven. Four of the catchment areas had a comprehensive range of services for this group of patients, including community-based admission facilities, respite care, long-term residential care, and day care. Two of these four catchment areas also had access to hospital-based admissions facilities but five catchment areas had no specialised facilities of any kind for the treatment of this group of patients, either in hospital or in the community. Informants in these areas reported that local services experience problems in managing acute psychiatric episodes among people with a learning disability, and need to refer patients to hospitals far from their home. This produces difficulties in maintaining contact between patients and their family and friends, and in providing continuity of care between hospital and community-based services.

At the time of the survey, all 25 catchment areas had some form of community mental handicap team or teams, although there were substantial variations in the number and range of professional staff involved. All 25 teams included consultants and community mental handicap nurses, with social workers represented on 21 teams, clinical psychologists on 17, occupational therapists on 15 , speech therapists on 11, and physiotherapists on nine teams. This diversity of membership patterns between CMHTs has been observed in a number of studies (Brown \& Wistow, 1990; Hudson, 1991).

\section{Developing a purchasing strategy}

The results of the survey indicate that several district health authorities in the West Midlands Health Region need to develop services for people with learning disabilities and additional psychiatric disorders as a matter of urgency. It is recommended that these services should meet the following guidelines.

They should provide a rapid response to crises, by a team of staff skilled in the diagnosis, treatment, and care of people with both a learning disability and a psychiatric disorder.

Wherever practicable, patients with a psychiatric disorder should be treated in their own home, with sufficient support given to primary carers.

Patients who require in-patient admission should be admitted to units that are locally accessible.

There should be close working relationships between local mental handicap and mental illness teams, and patients with mild learning disabilities who need acute admission because of a mental illness could be admitted to general psychiatric facilities where practicable.

There should be a suitable range of residential and day-care facilities for patients who have longterm disorders, as well as for those who require treatment for acute psychiatric episodes.

A model service to meet these guidelines should include a community team, with access to specialist admission facilities. The community team should comprise the relevant consultant psychiatrist and clinical psychologist for the district, together with nursing, social work, and paramedical staff skilled in dealing with both learning disabilities and mental illness. The aim of the team would be to provide assessment and intensive treatment for acute psychiatric disorders in the patient's home setting. This should include access to intensive day treatment facilities, which may be shared with those available to the local community mental health teams.

Where patients cannot be treated at home (usually because of a danger to others in the household), they should be admitted for acute treatment to a local inpatient psychiatric facility. Patients with a mild learning disability who have a functional psychiatric illness can often be managed in ordinary acute psychiatric wards, provided that staff on these wards have received additional training in dealing with this group of patients, and the ward team receives support from a psychiatrist with special skills in developmental psychiatry (NHS Management Executive, 1992). Patients who cannot be managed in ordinary acute psychiatric wards should be placed in an admission facility which specialises in the treatment of psychiatric disorders among people with a learning disability. The preferred location is on the site of general psychiatric facilities, although a site shared with other facilities for people with a learning disability would also be suitable. The key determining consideration should be whichever site is most locally accessible, most appropriately staffed, and has the strongest links with community services.

Patients with a learning disability and long-term and severe psychiatric and/or behavioural disorders who live in their own home or in ordinary supported housing require access to long-term psychiatric support. This can be provided by the local CMHT, provided it includes staff trained in work with this group of patients. The CMHT will require access to respite care and day care services which also have specialist skills in dealing with this group of patients. To provide for the small number of patients who cannot be managed in their own home or in ordinary supported housing, district health authorities need access to places in specialised supported housing. The latter 
should include 24 -hour staffing by staff trained in the management of severe behaviour disorders in people with a learning disability. Some health authorities have provided this kind of service in conversions of ordinary domestic houses, but this usually means very high staffing levels to maintain a staff rota for a small number of patients, and some houses may lack sufficient space for disturbed patients to act our their disorders. An alternative is to provide a unit of about ten beds, as part of a small cluster of specialist facilities for people with a learning disability, which would share day care and support costs. This could also provide specialised respite care for patients with longterm psychiatric disorders who live with their families.

Some health districts may be too small to provide the full range of these services. It may therefore be necessary for district health authorities to form consortia to purchase specialist psychiatric services for people with a learning disability from a single provider. It would facilitate liaison between the various services for people with a learning disability if such consortia had boundaries which corresponded to those of counties and metropolitan boroughs, and hence also of social services departments, education departments, and family health authorities.

Implementing a comprehensive local service of this kind requires a good working relationship between learning disability and mental illness services. These are often managed by separate management units in the same health authority, and any working relationships have usually developed informally between clinicians. Effective co-operation between these services should therefore be specified by district health authorities in setting contracts with both services.

\section{References}

Brown, S. \& Wistow, G. (1990) The Roles and Tasks of Community Mental Handicap Teams. London: Avebury. CoRbetr, J. A. (1979) Psychiatric morbidity and mental retardation. In Psychiatric Illness and Mental Handicap (eds. F. E. James and R. P. Snaith) London: Gaskell.

DEAN, C. \& GADD, E. (1990) Home treatment for acute psychiatric illness. British Medical Journal, 301, 10211023.

Hudson, R. (1991) A question of teamwork. Health Services Journal, 101,18-19.

LUND, J. (1985) The prevalence of psychiatric morbidity in mentally retarded adults. Acta Psychiatric Scandinavica, 72, 563-570.

NHS Management Executive (1992) HSG (92) 42 Health Services for People with Learning Disabilities.

A full list of references is available on request to Dr Cumella.

\section{COLLEGE SEMINARS SERIES}

\section{Seminars in Child and Adolescent Psychiatry}

\section{Edited by Dora Black \& David Cottrell}

This new series of books is intended to help junior doctors during their training years. Separate volumes will cover clinical psychiatry, each of its subspecialties, and also the relevant non-clinical academic disciplines of psychology and sociology. College Seminars will also make a contribution to the continuing medical education of established clinicians. Seminars in Child and Adolescent Psychiatry is the first volume to appear in the series. 